\title{
Managing diabetic foot in times of COVID-19: time to put the best 'foot' forward
}

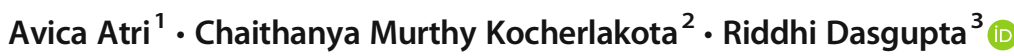 \\ Received: 29 June 2020 / Accepted: 19 August 2020 / Published online: 1 September 2020 \\ (C) Research Society for Study of Diabetes in India 2020
}

\begin{abstract}
Introduction The COVID-19 pandemic has had an unparalleled impact on the socio-economic and healthcare structure of India. Due to our large populations of diabetic patients, who have an increased risk of worse outcomes with COVID-19 infection, it is of utmost public health importance to analyse the relationship between the two. The aim of our review was to analyse the possible relationship between COVID-19 infection and DFUs, which are a fairly common, yet serious complication in diabetic patients, as well as their management, under the given changing circumstances.

Methodology An extensive review of related educational articles was analysed from various databases.

Results The two main pathogenic mechanisms described in COVID-19 infection are a cytokine storm (causing ARDS) as well as an acquired coagulopathy, with widespread thrombosis. DFUs are associated with an underlying peripheral neuropathy, a chronic low-grade inflammatory state and peripheral arterial disease, which lead to chronic non-healing ulcers. Similarities seen in the pathogenic mechanisms of these two conditions make a bidirectional relationship highly plausible.

Conclusion Due to the disruptions in the healthcare system brought on by the COVID-19 pandemic, changes in practice to a telehealth-driven approach, with emphasis on homecare and community clinics, need to be adopted, to ensure best possible care to patients with DFUs, in order to reduce their risk of DFU-related complications and need for hospitalization.
\end{abstract}

Keywords COVID-19 · Diabetic peripheral neuropathy (DPN) · Diabetic foot ulcers (DFU) · Cytokine storm · Ischemia

\section{Introduction}

The novel SARS-CoV-2 virus outbreak, which has developed into an unprecedented global pandemic, has massively impacted human health and economy worldwide. In India, at the time of writing this article, there were nearly 5 lakh infected cases, with more than 15,000 deaths [1], thus resulting in an unparalleled detrimental effect on the socio-economic and healthcare structure of the country.

Although a majority of the patients presenting with COVID-19 have been noted to have mild, self-resolving upper respiratory tract symptoms, with preservation of vital organ

Riddhi Dasgupta

riddhi_dg@rediffmail.com

1 Christian Medical College \& Hospital, Vellore, Tamil Nadu, India

2 Department of Diabetology, Dr. Mohan's Diabetes Specialities Centre, Chennai, Tamil Nadu, India

3 Department of Endocrinology, NM Hospitals, Kolkata, West Bengal 700008, India functions [2], it is potentially fatal in patients with comorbidities like diabetes, in whom it progresses to an acute respiratory distress syndrome (ARDS), resulting in it being a matter of major public health concern. Worse outcomes have been recorded in patients with comorbid conditions like diabetes mellitus, hypertension, chronic obstructive pulmonary disease, obesity and smoking [3-5].

India has one of the world's largest populations of diabetic patients, with the latter at an increased risk of developing worse outcomes following SARS-CoV-2 infection. Patients with diabetes are generally known to have an increased risk of infections, secondary to impaired immune responses, due to an altered cytokine profile, and $\mathrm{T}$ cell and macrophage activation, with poor glycemic control further increasing the risk [5]. In addition, diabetes is closely associated with obesity which adds to the risk of worse outcomes due to the development of a chronic low-grade inflammatory state, due to an abnormal secretion of adipokines and certain cytokines (TNF- $\alpha$ and interferons), that can further blunt antiviral responses [6]. Though the relationship of COVID-19 with diabetes has been extensively evaluated, its specific impact on the diabetic foot remains sparsely studied. 


\section{Diabetic foot and COVID-19: pathogenetic links}

Patients with diabetes develop various microvascular and macrovascular complications during their lifetime, of which peripheral neuropathy and diabetic foot ulcers (DFU) cause a significant negative impact on their quality of life. However, DFU still remains a widely under-reported complication, necessitating a thorough understanding of its etiopathogenesis in times of the COVID-19 pandemic.

\section{Exploring the inflammatory link}

There has been a growing clinical and experimental evidence to show the presence of chronic low-grade inflammation and elevated levels of inflammatory cytokines IL-1, 6, 18 and $\mathrm{TNF} \alpha$ to be involved in the pathogenesis of both diabetic peripheral neuropathy (DPN) [7] and in the development of DFU [8]. Data from animal studies further lend credence to the inflammatory influence on development of DPN, with diabetic mice treated with COX-2 inhibitors showing no neuropathy, as compared with an untreated group [9]. It is due to this complex mechanism of development of DPN that the treatment has proved to be challenging.

Diabetic foot ulcers develop as a consequence of peripheral neuropathy, abnormal foot mechanics, peripheral artery disease and poor wound healing [10], along with reduced blood flow secondary to peripheral arterial disease increasing the risk of secondary infection.

In diabetes, due to an unbalanced response between proand anti-inflammatory mediators, wound healing is impaired, leading to the development of chronic non-healing ulcers [11, 12]. Cytokine growth factors like PDGF, FGF, EGF, VEGF, IGF, TGF, etc. are said to be involved. Due to a disequilibrium studied in the levels of these cytokines, newer treatment modalities using various growth factors are being developed for chronic foot ulcers [13]. The three main factors hindering wound healing are peripheral neuropathy, peripheral arterial occlusive disease and superimposed infection. Also, abnormal or reduced expression of growth factors has been described in diabetic foot ulcers [14]. Some cytokines in the inflammatory cascade like IL-6 are considered to have dual effects, usually having been considered a pro-inflammatory cytokine is now being studied in the treatment of DPN, due to associated positive effects like enhanced blood flow, decreased chronic inflammation as well as peripheral nerve fibre regeneration [15].

The disease pathogenesis, as well as development of complications in SARS-CoV-2 infection, is believed to be via a cytokine storm, which is an uncontrolled aberrant systemic inflammatory response brought about by soluble proinflammatory chemical mediators produced by effector cells of the immune system [16]. In patients with COVID-19 infection, high blood levels of cytokines and chemokines have been reported, as in a study conducted by Huang et al. [17]. The cytokine storm, and its resultant ARDS, results from the effects of a combination of various molecules, of which interferons, interleukins, chemokines, colony-stimulating factors and TNF- $\alpha$ represent the main components involved in its pathogenesis [18]. Levels of various cytokines like IL-1 $\beta$, IL-6, IL-7, IL-10, Il-12, IL-13 and IFN- $\gamma$ have been shown to correlate severity in patients with SARS-CoV-2 infection [19]. In a study done by Jiang et al., chemokine CXCL10 levels proved to be a good prognostication index in patients with COVID-19 [20].

The SARS-CoV-2 virus is believed to infect cells via ACE2 receptors [21] of the respiratory tract, and as a result of which, diabetic patients who are on ACE inhibitors and ARBs have been shown to have an increased expression of ACE2 [22], increasing their risk of severe disease and possible fatality. DPN and chronic foot ulcers, which are influenced by abnormalities in cytokine levels, have similar pathogenic mechanisms as ARDS in SARS-CoV-2 infection, which is mainly brought about by a cytokine storm [16]. Thus, there seems to be a common inflammatory link between the two conditions.

Another unique manifestation of the diabetic foot is Charcot's neuroarthropathy (Charcot's foot). Secondary to the autonomic neuropathy, an increased local blood flow results in excessive bone resorption leading to a reduced bone mineral density. It is also associated with reduced sensation and impaired proprioception. This excessive local inflammation can result in local osteoporosis [23]. Recent studies have explored the central role of the RANKL-OPG pathway in the pathogenesis of Charcot's foot. A reduction in the neurons producing calcitonin gene-related peptide (CGRP) leads to an increase in RANKL with subsequent decline in joint integrity, thus aggravating the disease process [24]. Since the development and progression of Charcot's neuroarthropathy is closely linked with various inflammatory pathways, it is possible that a bidirectional relationship with COVID-19 infection may exist as well.

\section{Exploring the ischemic link}

Patients with DFU often have coexisting peripheral arterial disease (PAD) as a macrovascular complication [25]. Due to this, the occurrence of neuroischemic DFUs is on the rise, and timely intervention with revascularization can impact the ulcer progression timeline [26]. This association has been proved in various studies [26-29]. The neuroischemic component of DFUs is not only due to the macrovascular atherosclerosis but also as a result of impaired vasodilation secondary to the neuropathy, and as a result of which, even with correction of large vessel blood flow by successful bypass grafting surgery, the wound may not completely heal, although there may be considerable improvement [27]. This is the rationale behind 
all patients with DFUs requiring an Ankle-Brachial Index $(\mathrm{ABI})$ at baseline to quantify the degree of PAD.

Multiple observational studies have also shown an acquired condition, COVID-19-induced coagulopathy, with widespread development of arterial and venous thrombi in various vascular beds and with a multifactorial etiology [30]. Although the cause is still being widely studied, it has been seen to broadly follow Virchow's triad - endothelial damage, procoagulant substances in the circulation and altered blood flow. A study on 4 deceased patients in New Orleans showed small and firm thrombi in sections of the peripheral lung parenchyma, with no large thromboemboli at the hila [31]. In a study on 183 patients in Wuhan, China, D-dimer, fibrin degradation product (FDP) levels and prothrombin time (PT) were seen to have a significant correlation between disease severity [32]. The hypothesized ACE2-mediated entry of SARS-CoV-2 may be the means of endothelial injury and inflammation due to the widescale expression of ACE2 receptors on endothelial cells across various vascular beds [33]. The role of platelets and inflammatory cells like neutrophils in contributing to widespread microvascular thrombosis has been proposed via a mechanism involving neutrophil endothelial traps (NETs) that can activate the coagulation cascade via endothelial damage [30]. These pathophysiological observations have actually led to clinical recommendations for anticoagulation in COVID-19 patients.

Thus, COVID-19 infection has the propensity to worsen the neuro-ischemic component of DFUs. This has been corroborated in studies from Italy where increased occurrence of acute limb ischemia was seen in patients tested positive for COVID19 [34]. Given the procoagulant nature of COVID-19 infection, it is possible that patients with diabetic foot ulcers, who already have compromised vascular supply, could have worsening of their foot symptoms and can present with acute limbthreatening crisis requiring hospitalization. Such hospitalizations in diabetes patients with COVID-19 may in turn impact their chances of survival, thus setting up a vicious cycle.

\section{Exploring the vitamin D deficiency link}

The relationship between vitamin D deficiency, diabetic neuropathy and COVID-19 is another potentially exciting area of future research. Vitamin D deficiency has been associated with diabetes and its complications with lower levels of vitamin D seen in patients with DPN. In a study by Shehab et al., amongst 210 patients with diabetes, significantly lower levels of vitamin $\mathrm{D}(p<0.05)$ were seen amongst those with DPN and therefore proved to be an independent risk factor for DPN [35]. Also, reversal of chronic DPN has been shown with vitamin D supplementation in a deficient type 1 diabetes mellitus patient [36]. The pathogenesis of this phenomenon has been linked to deficient nerve growth in vitamin Ddeficient conditions [37]. Recent studies have focused on a possible link between COVID-19 and vitamin D deficiency, with suggestions that patients with vitamin D deficiency may have poorer outcomes than those without [38]. Since, vitamin $\mathrm{D}$ is involved in the production of antimicrobial substances in the respiratory tract [39], as well as in reducing inflammatory responses, its deficiency may pose an increased risk of worse outcomes with SARS-CoV-2 infection. Due to this possible dual beneficial effect, supplementing vitamin $\mathrm{D}$ in diabetic patients with DFUs and COVID-19 needs to be further looked into as a therapeutic modality.

Figure 1 enumerates the pathogenetic links described above.

\section{Management}

Diabetic foot ulcers alone are associated with an increased mortality in diabetic patients (nearly 2.5 times) [40], which can be reduced with adequate treatment and follow-up. In the times before the COVID-19 pandemic, wound care largely aimed at healing patient wounds with a combination of judicious antibiotics, adequate dressing and appropriate offloading. Since these require regular hospital visits, the goal of management has shifted focus to preventing development of wound complications and hospitalization in COVID-19 times [41]. In spite of the pandemic-induced disruption of local practices, it is essential that these patients continue receiving appropriate care while minimizing their need to visit the hospital, and the aim of the physician should be to shift focus away from hospital-based care [42]. Based on the International Working Group on the Diabetic Foot (IWGDF) guidelines [43], most patients with diabetic foot disease do not require hospitalization, unless they have severe infection with possible sepsis or require surgical intervention. By triaging patients, most can be managed as outpatients-with homecare, telemedicine appointments where possible and by setting up clinics at other locations outside of the hospital, with assistance as required from multidisciplinary diabetic foot clinics. Due to the increased susceptibility of this patient group to COVID-19, it is important to ensure that they practice social distancing and hand hygiene while visiting the designated diabetes foot clinics. Also, there is a need to emphasize the importance of proper glycemic control, which may be affected by COVID-19-induced restrictions on outdoor exercise activities. The patients also need to be reminded to continue using their offloading devices or special footwear at home, which may be harder during extended periods of lockdown. The importance of continued exercise as well as any other psychological needs should be catered to via telemedicine appointments. Since physicians will not be able to see their patients in person as often, diagnosing infection can be challenging. A thorough history eliciting important aspects of the temporal profile of ulcer development, features of local 


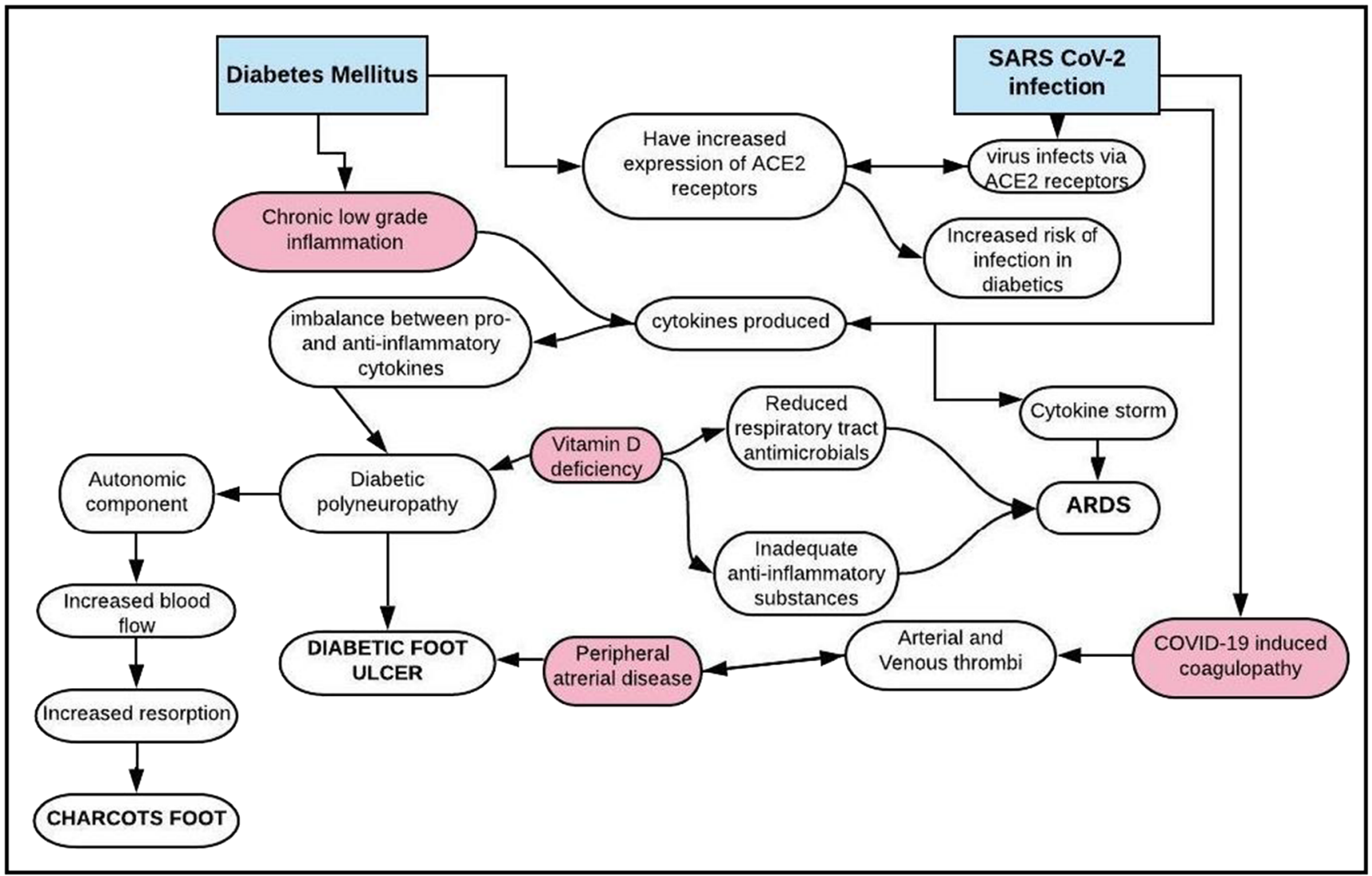

Fig. 1 Pathogenesis: diabetes mellitus, DFUs and COVID-19

and systemic inflammation, wound discharge and glycemic control can help the clinician in this aspect, and antibiotics, if needed, can be prescribed. Patients and their caretakers should be educated on checking the feet for infection or other signs of wound deterioration using handheld mirrors. Education regarding adequate feet hygiene, nail care, appropriate selection of socks and footwear can be imparted over telecommunication and can significantly mitigate the risk of developing DFU. Further, patients should be provided with detailed information on whom to contact and which hospital to go in case any ulcer complication develops.

Effective offloading is known to accelerate ulcer healing and reduce the infection rates and need for hospitalization. Thus, the reduced need for frequent ulcer debridement and dressings can help reduce clinic visits, especially during these times. Although the preferred choice for offloading includes non-removable offloading devices, as patients will not be able to make as many visits to the hospital, the use of removable offloading devices can be promoted. Interestingly, due to the predominantly respiratory symptoms of COVID-19 infection, patients may be less ambulant, and it is possible that this may help with ulcer offloading and accelerate ulcer healing [44]. Emphasis on proper foot care directly reduces their need to visit a healthcare centre and in turn reducing their chance of acquiring COVID infection.
Diagnosis of associated peripheral arterial disease may need to be done over video calls due to the inability to see patients in person. This can be done by analysing certain features like rest pain at the wound while lying flat, tissue loss with new dry or wet gangrene that may mandate urgent surgical debridement or the Buerger test for arterial insufficiency (blanching, numbness and pain at the extremity that develops within a few minutes of limb elevation and reduces when the leg is lowered below horizontal level). If hemodynamic tests are available, critical ischemia will be indicated by ankle systolic pressure $<50 \mathrm{mmHg}$ and toe systolic pressure $<$ $30 \mathrm{mmHg}$ [45]. In patients found to have associated limbthreatening ischemia, the benefit of revascularization to help wound healing and limb salvage outweighs the risks of acquiring COVID-19 infection and is therefore the advisable course of action. The Wound Care Centre Without Walls model of dealing with chronic wound patients during this pandemic is gaining popularity, in order to continue providing wound care to patients untethered to the hospital setting but by using technology and community centred care [41].

Amputations are often required to save the life of affected patients with diabetic foot ulcers that have associated irreversible limb, with severe vascular disease or superimposed ischemic infection. These should be performed after alternative treatments are extensively considered, in view of the high risk 
of disease transmission by COVID-19 infected patients during surgery, and should be considered only in patients who will physically be able to tolerate surgery - in a designated hospital, with the required protective equipment. In a patient who presents with severe symptoms of COVID-19, with indications for surgery, the main target should be survival [46]. While optimizing patient care in such scenarios can be challenging, utmost care should also be taken to reduce the threat of infection to the attending surgeons, medical practitioners, nurses and associated healthcare workers.

Based on the guidelines by the American College of Surgeons, guidelines for surgical procedures have been described in Table 1 [41].

\section{COVID-19 and diabetic foot: challenges in the Indian scenario}

While most of these guidelines can be applied reasonably well in the western countries, in India, putting many of these guidelines to practice is a challenge due to multiple socio-economic, demographic and healthcare-related diversities across the country. Due to the generally low health-seeking behavior, most patients with diabetic foot ulcers do not realize the need for supervised care, more so amongst those in the lower socioeconomic strata, for whom access to proper healthcare is almost always out of reach. This apprehensive behavior may aggravate during pandemics like COVID-19, with initial neglect of a superficial ulcer leading to severe infections and unnecessary hospitalizations, which in turn can spread the pandemic more rapidly. This is where widespread use of telemedicine can be of vital importance. Various government organizations have established telemedicine linkages from many rural areas to hospitals in nearby cities, which in these COVID-19 times can be of immense use, especially for patients with chronic conditions like diabetic foot. Based on the guidelines by the Ministry of Health and Family Welfare (MOHFW) [47], state-registered medical practitioners are eligible to practice using telemedicine which provides physicians with the opportunity to continue managing their patients during this COVID-19 pandemic, except in a few instances where patients need to be seen in person or if hospitalization is required [48]. Wherever possible, the use of a two-way video communication means (like WhatsApp, Facetime, Zoom, etc.) can help the physician in visual evaluation of the wounds and prescribing empirical antibiotics and offloading. Patients can then be asked to send back a photo of their ulcer once a week to the podiatry team, to follow up any improvement or deterioration in their condition. However, telehealth may not suffice in complicated wounds or ischemic foot ulcers and require inperson evaluation. Here also, depending on where the local healthcare system is in the pandemic curve, podiatric nurses and healthcare workers previously caring for patients in the foot care facility can be pivoted to communicating with patients, families and home health services to evaluate the best plan of care, with the goal of keeping the patient at home whenever possible.

Dressing and offloading devices provided by a reliable medical supplier are of paramount importance to sustain home-based care during the pandemic. Dressings in singledose packages, use of easily available, low-cost materials (like Samadhanam dressing with roller-gauze) [49] and video tutorials on dressing changes are innovative ways that can be adopted in these times.

Table 1 Approach to wound-related surgical procedures in the COVID-19 pandemic

\begin{tabular}{lll}
\hline Category & Condition & Management tier \\
\hline \multirow{3}{*}{ Wounds } & Lower limb disease with non-salvageable limb (amputation) & 3 Do not postpone \\
Gangrene & Amputation for infection/necrosis (TMA, BKA, AKA) & 3 Do not postpone \\
Amputation & Amputation for necrosis/infection of toes & $2 \mathrm{~b}$ Postpone if possible \\
& Deep debridement of surgical wound infection or necrosis & $2 \mathrm{~b}$ Postpone if possible \\
Venous & Wounds requiring skin grafts & 2b Postpone if possible \\
& Procedures for ulceration secondary to venous disease & 2a Consider postponing \\
& Acute limb ischaemia & 3 Do not postpone \\
Peripheral & Limb ischaemia, progressive tissue loss, acute limb ischemia, & 3 Do not postpone \\
Vascular disease & wet gangrene, ascending cellulitis & 3 Do not postpone \\
& Fasciotomy for compartment syndrome & $2 b$ Postpone if possible \\
& Peripheral vascular disease: chronic limb threatening ischemia, & 2b Postpone if possible \\
& rest pain or tissue loss & 1 Postpone \\
\hline
\end{tabular}

TMA Transmetatarsal amputation, $B K A$ below-knee amputation, $A K A$ above-knee amputation

Adapted from the 'Wound Center Without Walls: The New Model of Providing Care During the COVID-19 Pandemic' 


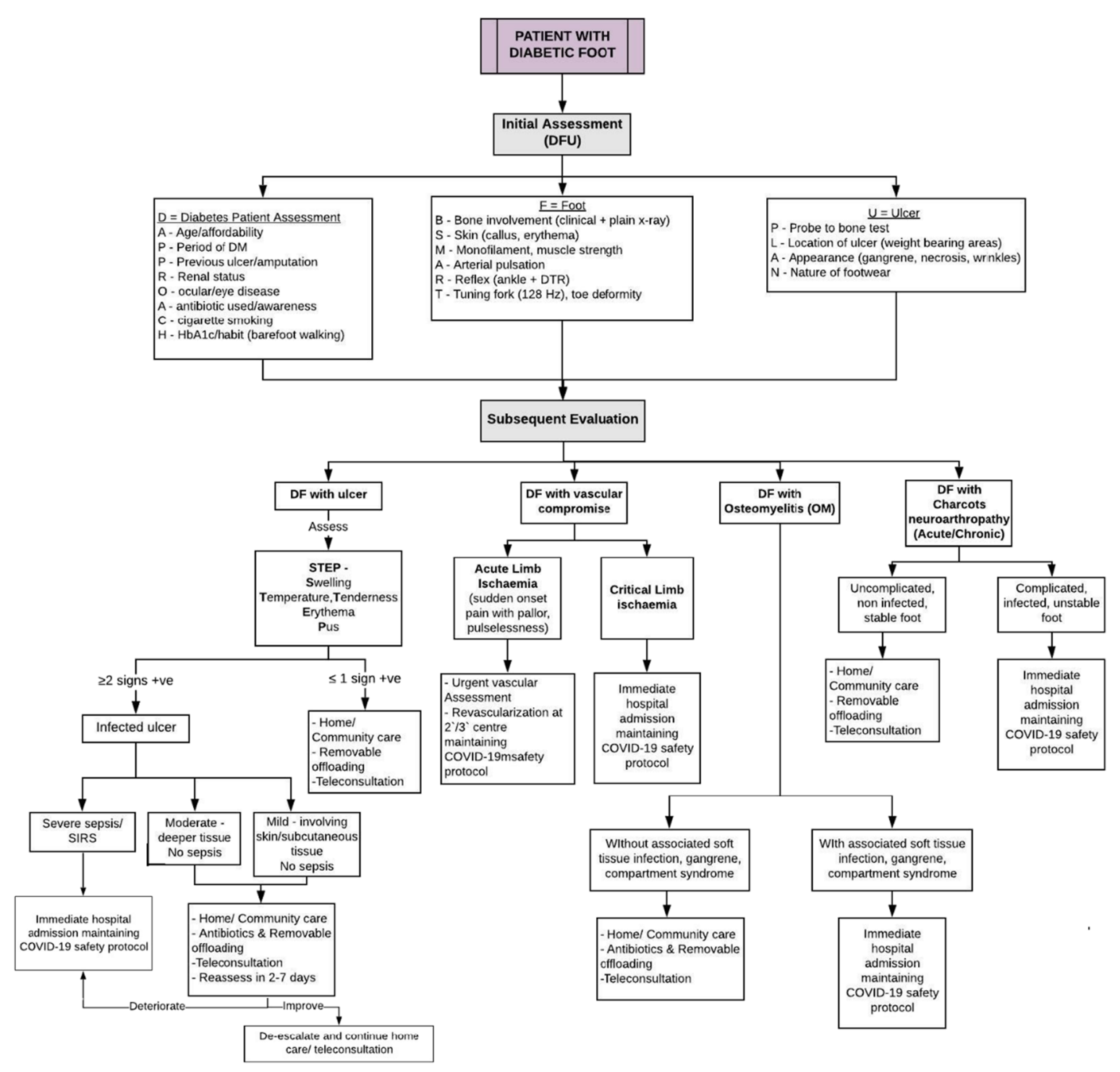

Abbreviations:

DM - Diabetes Mellitus; DF - Diabetic Foot; DFU - Diabetic Foot Ulcer;

SIRS - Systemic Inflammatory Response Syndrome; DTR - Deep Tendon Reflexes

Fig. 2 Approach to management of diabetic foot care in the COVID-19 pandemic in India

In patients with complicated DFU who require a clinic or hospital visit, novel strategies including shifting the foot care facility to a medical office building outside the physical space of the COVID hospital, reducing crowding in the waiting area and expediting the evaluation and treatment of wounds can be implemented. Providing home-based intravenous antibiotics and early switchover to oral antibiotics are simple measures that can reduce the burden on the healthcare system which is already being stretched to capacity by the pandemic.

\section{Conclusion}

The COVID-19 pandemic has brought about healthcare disruptions of unprecedented magnitude, with diabetes patients bearing the brunt of the infection globally. Diabetic foot presents a clinical conundrum, where ingenious solutions are needed to provide optimal wound care while minimizing the exposure risk. Patients with DFU often have multiple comorbidities and fit several of the high-risk criteria for COVID-19 infection. Rather than avoiding these patients, it is imperative 
to devise government-approved, telehealth-driven structured algorithms to treat them effectively. Since most existing international guidelines $[43,50]$ are suited to the western population, a modified triage and treatment algorithm has been suggested by the authors which may be (Fig. 2) more apt in tackling the dual menace of diabetic foot and COVID-19 in the Indian population.

\section{Compliance with ethical standards}

Conflicts of interest The authors declare that they have no conflict of interest.

\section{References}

1. 20200607-covid-19-sitrep-139.pdf [Internet]. [cited 2020 Jun 8]. Available from: https://www.who.int/docs/default-source/ coronaviruse/situation-reports/20200607-covid-19-sitrep-139.pdf? sfvrsn $=79 \mathrm{dc} 6 \mathrm{~d} 082$

2. Gupta N, Agrawal S, Ish P, Gaind R, Arora B, Sen M, et al. Clinical and epidemiologic profile of the initial COVID 19 patients at a tertiary care centre in India. Monaldi archives for chest disease = Archivio Monaldi per le malattie del torace / Fondazione clinica del lavoro, IRCCS [and] Istituto di clinica tisiologica e malattie apparato respiratorio, Università di Napoli. Secondo Ateneo. 2020;90:193-6.

3. Guan W, Liang W, Zhao Y, Liang H, Chen Z, Li Y, et al. Comorbidity and its impact on 1590 patients with COVID-19 in China: a nationwide analysis. Eur Respir J [Internet]. 2020 May 14 [cited 2020 Jun 5];55(5). Available from: https://www.ncbi.nlm. nih.gov/pmc/articles/PMC7098485/

4. Solís P, Carreňo H. COVID-19 fatality and comorbidity risk factors among confirmed patients in Mexico [Internet]. Epidemiology; 2020 Apr [cited 2020 Jun 5]. Available from: http://medrxiv.org/ lookup/doi/10.1101/2020.04.21.20074591

5. COVID-19 infection in people with diabetes touchENDOCRINOLOGY [Internet]. [cited 2020 Jun 5]. Available from: https://www.touchendocrinology.com/insight/ covid-19-infection-in-people-with-diabetes/

6. Honce R, Schultz-Cherry S. Impact of obesity on influenza A virus pathogenesis, immune response, and evolution. Front Immunol. 2019;10:1071.

7. Navarro-González JF, Mora-Fernández C. The role of inflammatory cytokines in diabetic nephropathy. JASN. 2008;19(3):433-42.

8. Jin HY, Park TS. Role of inflammatory biomarkers in diabetic peripheral neuropathy. J Diabetes Investig. 2018;9(5):1016-8.

9. Kellogg AP, Wiggin TD, Larkin DD, Hayes JM, Stevens MJ, PopBusui R. Protective effects of cyclooxygenase-2 gene inactivation against peripheral nerve dysfunction and intraepidermal nerve fiber loss in experimental diabetes. Diabetes. 2007;56(12):2997-3005.

10. Diabetic foot ulcers: pathogenesis and prevention - clinical correlations [Internet]. [cited 2020 Jun 6]. Available from: https://www. clinicalcorrelations.org/2015/03/19/diabetic-foot-ulcerspathogenesis-and-prevention/

11. Falanga V. Wound healing and its impairment in the diabetic foot. Lancet. 2005;366(9498):1736-43.

12. Pop-Busui R, Ang L, Holmes C, Gallagher K, Feldman EL. Inflammation as a therapeutic target for diabetic neuropathies. Curr Diab Rep. 2016;16(3):29.
13. Zubair M, Ahmad J. Role of growth factors and cytokines in diabetic foot ulcer healing: a detailed review. Rev Endocr Metab Disord. 2019;20(2):207-17.

14. Papanas N, Maltezos E. Growth factors in the treatment of diabetic foot ulcers: new technologies, any promises? Int J Lower Extr Wound. 2007;6(1):37-53.

15. Cox AA, Sagot Y, Hedou G, Grek C, Wilkes T, Vinik AI, et al. Low-dose pulsatile interleukin-6 as a treatment option for diabetic peripheral neuropathy. Front Endocrinol (Lausanne) [Internet]. 2017 May 2 [cited 2020 Jun 14];8. Available from: https://www. ncbi.nlm.nih.gov/pmc/articles/PMC5411416/

16. Nile SH, Nile A, Qiu J, Li L, Jia X, Kai G. COVID-19: Pathogenesis, cytokine storm and therapeutic potential of interferons. Cytokine Growth Factor Rev [Internet]. 2020 May 7 [cited 2020 Jun 6]; Available from: https://www.ncbi.nlm.nih.gov/pmc/ articles/PMC7204669/

17. Huang C, Wang Y, Li X, Ren L, Zhao J, Hu Y, et al. Clinical features of patients infected with 2019 novel coronavirus in Wuhan, China. Lancet. 2020;395(10223):497-506.

18. Coperchini F, Chiovato L, Croce L, Magri F, Rotondi M. The cytokine storm in COVID-19: an overview of the involvement of the chemokine/chemokine-receptor system. Cytokine Growth Factor Rev. 2020;53:25-32.

19. Costela-Ruiz VJ, Illescas-Montes R, Puerta-Puerta JM, Ruiz C, Melguizo-Rodríguez L. SARS-CoV-2 infection: the role of cytokines in COVID-19 disease. Cytokine Growth Factor Rev [Internet]. 2020 Jun 2 [cited 2020 Jun 11]; Available from: https://www.ncbi.nlm.nih.gov/pmc/articles/PMC7265853/

20. Jiang Y, Xu J, Zhou C, Wu Z, Zhong S, Liu J, et al. Characterization of cytokine/chemokine profiles of severe acute respiratory syndrome. Am J Respir Crit Care Med. 2005;171(8): $850-7$.

21. Wan Y, Shang J, Graham R, Baric RS, Li F. Receptor recognition by the novel coronavirus from Wuhan: an analysis based on decade-long structural studies of SARS coronavirus. J Virol. 2020;17:94(7).

22. Li XC, Zhang J, Zhuo JL. The vasoprotective axes of the reninangiotensin system: physiological relevance and therapeutic implications in cardiovascular, hypertensive and kidney diseases. Pharmacol Res. 2017;125(Pt A):21-38.

23. Papanas N, Maltezos E. Etiology, pathophysiology and classifications of the diabetic Charcot foot. Diabetic Foot \& Ankle. 2013;4(1):20872.

24. Jeffcoate WJ. Charcot neuro-osteoarthropathy. Diabetes Metab Res Rev. 2008;24(S1):S62-5.

25. Armstrong DG, Cohen K, Courric S, Bharara M, Marston W. Diabetic foot ulcers and vascular insufficiency: our population has changed, but our methods have not. J Diabetes Sci Technol. 2011;5(6):1591-5.

26. Khan Y, Khan MM, Jain A, Namdev RK. A study of association of diabetic foot ulcers and peripheral vascular disease. Int J Adv Med. 2018;5(6):1454-9.

27. Arora S, Pomposelli F, LoGerfo FW, Veves A. Cutaneous microcirculation in the neuropathic diabetic foot improves significantly but not completely after successful lower extremity revascularization. J Vasc Surg. 2002;35(3):501-5.

28. Adler AI, Boyko EJ, Ahroni JH, Smith DG. Lower-extremity amputation in diabetes. The independent effects of peripheral vascular disease, sensory neuropathy, and foot ulcers. Diabetes Care. 1999;22(7):1029-35.

29. Tresierra-Ayala MÁ, García RA. Association between peripheral arterial disease and diabetic foot ulcers in patients with diabetes mellitus type 2. Medicina Universitaria. 2017;19(76):123-6.

30. Becker RC. COVID-19 update: Covid-19-associated coagulopathy. J Thromb Thrombolysis. 2020;15:1-14. 
31. Pulmonary and cardiac pathology in Covid-19: the first autopsy series from New Orleans | medRxiv [Internet]. [cited 2020 Jun 13]. Available from: https://www.medrxiv.org/content/10. 1101/2020.04.06.20050575v1

32. Tang N, Li D, Wang X, Sun Z. Abnormal coagulation parameters are associated with poor prognosis in patients with novel coronavirus pneumonia. J Thromb Haemost. 2020;18(4):844-7.

33. Monteil V, Kwon H, Prado P, Hagelkrüys A, Wimmer RA, Stahl $\mathrm{M}$, et al. Inhibition of SARS-CoV-2 infections in engineered human tissues using clinical-grade soluble human ACE2. Cell. 2020 14;181(4):905-913.e7.

34. Bellosta R, Luzzani L, Natalini G, Pegorer MA, Attisani L, Cossu LG, et al. Acute limb ischemia in patients with COVID-19 pneumonia. J Vasc Surg [Internet]. 2020 Apr 29 [cited 2020 Jun 6]; Available from: https:/www.ncbi.nlm.nih.gov/pmc/articles/ PMC7188654/

35. Shehab D, Al-Jarallah K, Mojiminiyi OA, Al Mohamedy H, Abdella NA. Does vitamin D deficiency play a role in peripheral neuropathy in type 2 diabetes? Diabet Med. 2012;29(1):43-9.

36. Bell DSH. Reversal of the symptoms of diabetic neuropathy through correction of vitamin D deficiency in a type 1 diabetic patient. Case Rep Endocrinol [Internet]. 2012 [cited 2020 Jun 13];2012. Available from: https:/www.ncbi.nlm.nih.gov/ pmc/articles/PMC3530756/

37. Riaz S, Malcangio M, Miller M, Tomlinson DR. A vitamin $\mathrm{D}(3)$ derivative (CB1093) induces nerve growth factor and prevents neurotrophic deficits in streptozotocin-diabetic rats. Diabetologia. 1999;42(11):1308-13.

38. Mitchell F. Vitamin-D and COVID-19: do deficient risk a poorer outcome? Lancet Diabetes Endocrinol. 2020;S2213858720301832.

39. Gombart AF. The vitamin D-antimicrobial peptide pathway and its role in protection against infection. Future Microbiol. 2009;4:115165 .

40. Walsh JW, Hoffstad OJ, Sullivan MO, Margolis DJ. Association of diabetic foot ulcer and death in a population-based cohort from the United Kingdom. Diabet Med. 2016;33(11):1493-8.

41. Rogers LC, Armstrong DG, Capotorto J, Fife CE, Garcia JR, Gelly $\mathrm{H}$, et al. Wound center without walls: the new model of providing care during the COVID-19 pandemic. Wounds. 2020;24.
42. Rogers LC, Lavery LA, Joseph WS, Armstrong DG. All feet on deck-the role of podiatry during the COVID-19 pandemic: preventing hospitalizations in an overburdened healthcare system, reducing amputation and death in people with diabetes. J Am Podiatr Med Assoc. 2020;25.

43. COVID-19 and diabetic foot disease [Internet]. IWGDF Guidelines. [cited 2020 Jun 8]. Available from: https:// iwgdfguidelines.org/covid-19/

44. Papanas N, Papachristou S. COVID-19 and diabetic foot: will the lamp burn bright?: The International Journal of Lower Extremity Wounds. 2020 Jun 1;19(2):111-111. https://doi.org/10.1177/ 1534734620921382

45. Diabetes foot care in the COVID-19 pandemic [Internet]. D-Foot. [cited 2020 Jun 8]. Available from: https://www.d-foot.org/ resources/columns/feet-first/diabetes-foot-care-in-the-covid-19pandemic

46. Tao F, Tang X, Tao H, Luo Y, Cao H, Xiang W, et al. Surgical treatment of diabetic foot ulcers during the COVID-19 pandemic in China. J Diabetes Complicat. 2020;14:107622.

47. Telemedicine.pdf [Internet]. [cited 2020 Jun 8]. Available from: https://www.mohfw.gov.in/pdf/Telemedicine.pdf

48. Ghosh A, Gupta R, Misra A. Telemedicine for diabetes care in India during COVID-19 pandemic and national lockdown period: guidelines for physicians. Diabetes Metab Syndr. 2020;14(4):273-6.

49. Dhivya S, Padma VV, Santhini E. Wound dressings - a review. Biomedicine (Taipei) [Internet]. 2015 Nov 28 [cited 2020 Jun 26];5(4). Available from: https://www.ncbi.nlm.nih.gov/pmc/ articles/PMC4662938/

50. Lipsky BA, Berendt AR, Cornia PB, Pile JC, Peters EJG, Armstrong DG, et al. 2012 Infectious Diseases Society of America Clinical Practice Guideline for the Diagnosis and Treatment of Diabetic Foot Infections. Clin Infect Dis. 2012;54(12):e132-73.

Publisher's note Springer Nature remains neutral with regard to jurisdictional claims in published maps and institutional affiliations. 